\title{
Experimental investigations of the interface between steam and water two phase flows
}

\author{
Afrasyab Khan ${ }^{\mathrm{a}, *}$, Noaman Ul Haq ${ }^{\mathrm{a}}$, Imran Rafiq Chughtai ${ }^{\mathrm{b}}$, Ajmal Shah ${ }^{\mathrm{c}}$, Khairuddin Sanaullah ${ }^{\mathrm{d}}$ \\ a School of Chemical and Materials Engineering (SCME), Department of Chemical Engineering, National University of Sciences and Technology (NUST), H-12-Islamabad, Pakistan \\ ${ }^{\mathrm{b}}$ Department of Chemical Engineering, Pakistan Institute of Engineering and Applied Sciences (PIEAS), Nilore, Islamabad, Pakistan \\ ${ }^{\mathrm{C}}$ Department of Mechanical Engineering, Pakistan Institute of Engineering and Applied Sciences (PIEAS), Nilore, Islamabad, Pakistan \\ ${ }^{\mathrm{d}}$ Department of Chemical Engineering and Energy Sustainability, University Malaysia Sarawak (UNIMAS), Malaysia
}

\section{A R T I C L E I N F O}

\section{Article history:}

Received 13 September 2013

Received in revised form 13 February 2014

Accepted 13 February 2014

Available online 14 March 2014

\section{Keywords:}

Pressure

Hydrodynamic

Instability

Temperature

Steam

Water

\begin{abstract}
A B S T R A C T
Hydrodynamic instabilities play an important role in the design of those systems operated at high temperature and pressure. The creation and propagation of Kelvin-Helmholtz $(\mathrm{KH})$ instabilities has been experimentally investigated here for the first time in condensable fluids i.e. steam and water. Generally, in case of these condensable fluids the instabilities are so much short lived that it's very difficult to record them. Here the instabilities occurred in the close vicinity of the steam-water interface, were attributed mainly to the temperature fluctuations of micro scale or less. Supersonic steam was injected inside the subcooled water at inlet pressure varying from 1.5 bar to 3.0 bar by using a specially designed supersonic nozzle, whereas temperature of water inside the vessel was raised from $30^{\circ} \mathrm{C}$ to $60^{\circ} \mathrm{C}$ at an increment of 50 each. Kelvin-Helmholtz instabilities in the form of minor as well as amplified transient temperature fluctuations were recorded using a specially designed apparatus that uses LM35 sensors and data acquisition system. This system is capable to record temperatures at a rate of one millisecond and was also capable to record the temperatures anywhere inside the vessel. It was also found out that the instabilities created at the interface propagated towards the axis of the geometry as well as these instabilities were strongly affected by change in tank water temperature and inlet pressure.
\end{abstract}

(c) 2014 Elsevier Ltd. All rights reserved.

\section{Introduction}

Injection of steam jet into subcooled water tank has been the subject of study for the last two decades because of its numerous applications in nuclear and process industry. When steam and water come into direct contact with each other, an interface occurs between the two phases. The phenomenon of steam water interaction becomes very complicated due to the existence of this steam water interface in such flows. The behavior of steam-water interface is difficult to be captured theoretically, experimentally and/ or computationally because of its unstable nature. In addition to having unstable nature, occurrence of a transport of heat, mass and momentum across this interface makes the process more complicated.

The study of steam water interface is important because the unstable nature of the interface may result into the formation of

\footnotetext{
* Corresponding author.

E-mail addresses: afrasyab@scme.nust.edu.pk (A. Khan), noaman@scme.nust. edu.pk (N.U. Haq), imran@pieas.edu.pk (I.R. Chughtai), fac279@pieas.edu.pk (A. Shah), skhairuddin@feng.unimas.my (K. Sanaullah).
}

hydrodynamic instabilities and the condensation of steam across the interface on macro scale may lead to condensation induced water hammer inside the piping system of a nuclear power plant. The hydrodynamic instability at steam water interface along with water hammering due to condensation may cause a severe impact on the safe operation of nuclear power plant, especially under accidental conditions. Therefore, a detailed study is required to fully understand the behavior of steam-water interface.

The hydrodynamic instabilities in Direct Contact Condensation (DCC) are generally short lived and damped by the surrounding water. As a result the pressure and temperature shocks transform into hydrodynamic fluctuations of micro scale. The interface, at which these instabilities emerge and propagate, is considered a zero thickness surface as discussed by Shah et al. [1]. The fluctuations that occur at the interface and its vicinity are so much higher in amplitude, short lived and complicated that these are almost impossible to be modeled, simulated and difficult to observe experimentally. Therefore, till the date, the theoretical, computational and or experimental efforts to investigate the hydrodynamic instabilities formation and propagation particularly within condensable fluids are scarce. However, the interfacial instabilities of 